\title{
Exploring Reconfigurable Intelligent Surfaces for 6G: State-of- the-Art and the Road Ahead
}

This paper was downloaded from TechRxiv (https://www.techrxiv.org).

\section{LICENSE}

CC BY 4.0

SUBMISSION DATE / POSTED DATE

24-02-2022 / 03-03-2022

\section{CITATION}

Basharat, Sarah; Khan, Maryam; Iqbal, Muhammad; Hashmi, Umair; Zaidi, Syed Ali Raza; Robertson, Ian (2022): Exploring Reconfigurable Intelligent Surfaces for 6G: State-of-the-Art and the Road Ahead. TechRxiv. Preprint. https://doi.org/10.36227/techrxiv.19230138.v1

$\mathrm{DOI}$

10.36227/techrxiv.19230138.v1 


\title{
Exploring Reconfigurable Intelligent Surfaces for 6G: State-of-the-Art and the Road Ahead
}

\author{
Sarah Basharat, Maryam Khan, Muhammad Iqbal, Umair Sajid Hashmi, Member, IEEE, \\ Syed Ali Raza Zaidi, Member, IEEE, Ian Robertson, Fellow, IEEE
}

\begin{abstract}
Reconfigurable intelligent surfaces (RISs) are envisioned to transform the propagation space into a smart radio environment (SRE) to realize the diverse applications of sixthgeneration (6G) wireless communication. By smartly tuning the massive number of elements via controller, an RIS can passively phase-shift the electromagnetic (EM) waves to enhance the system performance. The absence of radio-frequency (RF) chains makes RIS an energy-efficient and cost-effective solution for future wireless networks. In this paper, we explore the state-of-theart research on different aspects of RIS-assisted communication. Specifically, we first introduce the fundamentals of RIS, including the RIS's structure, operating principle, and deployment strategies. We then comprehensively discuss the emerging applications of RISs for 6G wireless networks. In addition, we elaborate on the crucial challenges for RIS-assisted networks, namely, RIS channel state information (CSI) acquisition and passive beamforming optimization. Furthermore, we present the recent research contributions leveraging the artificial intelligence (AI) based techniques for channel estimation, phase-shift optimization, and resource allocation in RIS-assisted networks. Finally, to provide effective guidance for future research, we highlight important research directions for realizing RIS-assisted network.
\end{abstract}

\section{INTRODUCTION}

The demand for wireless capacity is continuously growing with the advent of Internet of Everything (IoE) system, connecting millions of people and billions of machines [1], [2]. According to a report from Cisco, the global mobile traffic volume is anticipated to grow to 5016 exabytes/month (EB/mo) by 2030 with a $65 x$ increase over 2023 [3]. To date, the fifth generation $(5 \mathrm{G})$ wireless networks are being rolled out in the world, providing a new vision to mobile communications [4]. The performance enhancement brought by the key $5 \mathrm{G}$ technologies, massive multiple-input multiple-output (MIMO) and millimeter-wave (mmWave) communications are undeniable. While mmWave communication promises to enhance the data rates by tapping in the yet under-utilized immense bandwidth at higher frequency spectrum [5], on the other hand, massive MIMO employs very large antenna arrays to reduce the intra-cell interference using simple signal processing techniques [6]. These technological advancements can be complemented with novel cell design architectures, such as

S. Basharat, M. Khan, M. Iqbal and U. S. Hashmi are with the School of Electrical Engineering and Computer Science (SEECS), National University of Sciences and Technology (NUST), Islamabad 44000, Pakistan (email: sbasharat.msee19seecs@ seecs.edu.pk; mkhan.msee19seecs@seecs.edu.pk; miqbal.msee19seecs@seecs.edu.pk; umair.hashmi@seecs.edu.pk).

S. A. R. Zaidi and I. Robertson are with the School of Electronic and Electrical Engineering, University of Leeds, United Kingdom (email: S.A.Zaidi@leeds.ac.uk; i.d.robertson@leeds.ac.uk). user-centric topologies supported through cloud radio access networks, to enhance user experience [7], [8]. However, these improvements are at the cost of complex hardware and high energy consumption. Moreover, the vulnerability of mmWave to signal blockages aggravates the situation, rendering these technologies insufficient on their own to meet the requirements for future wireless communication. Yet another limitation of these technologies is their incapability of controlling the wireless channel, which adversely affects the performance. While $5 \mathrm{G}$ is still in its commercialization phase, the research on sixth generation $(6 \mathrm{G})$ communication has already started ground work on innovative technologies that can support the capacity growth of future networks with lower cost, energy consumption, and hardware complexity. In this regard, a new concept of smart radio environments (SREs) has emerged [9], with the capability to tune the propagation channel according to the use case application. In order to realize the SREs, a disruptive technology, reconfigurable intelligent surface (RIS), also called intelligent reflecting surface (IRS), has attracted the research community [10], [11], [12]. RISs are envisioned to be capable of transforming the dumb wireless environment into an intelligent and smart space that can play an active role in improving the quality-of-service (QoS) and connectivity.

Specifically, an IRS is a two-dimensional planner surface consisting of a massive number of low-cost passive reflecting elements [12]. Each element can tune the phase of the incident signal to create a favorable propagation environment between the transmitter and the receiver. An RIS is not only conceptually appealing but also offers great advantages for practical implementation, as it does not require energy hungry sources, i.e., radio-frequency (RF) chains. The absence of RF chains makes RIS an energy efficient and cost effective solution as compared to the massive MIMO technology, which requires an RF chain for each antenna element [13], consequently increasing the hardware cost, complexity, and power consumption.

Different from the existing technologies, RIS has the potential to realize the diverse applications of $6 \mathrm{G}$ at low hardware cost and energy consumption. As illustrated in Fig. 1, RIS can also be integrated with the emerging communication technologies, such as non-orthogonal multiple access (NOMA), thus improving connectivity and coverage range [14], [15], [16], [17]. RIS can mitigate the signal blockage in mmWave and terahertz (THz) communications [18], [19], [20], [21], [22]. In mobile edge computing (MEC) networks, integration of RIS technology can improve the latency performance [23], [24], [25], [26], [27], [28]. Similarly, RIS can compensate for the significant power in simultaneous wireless information 


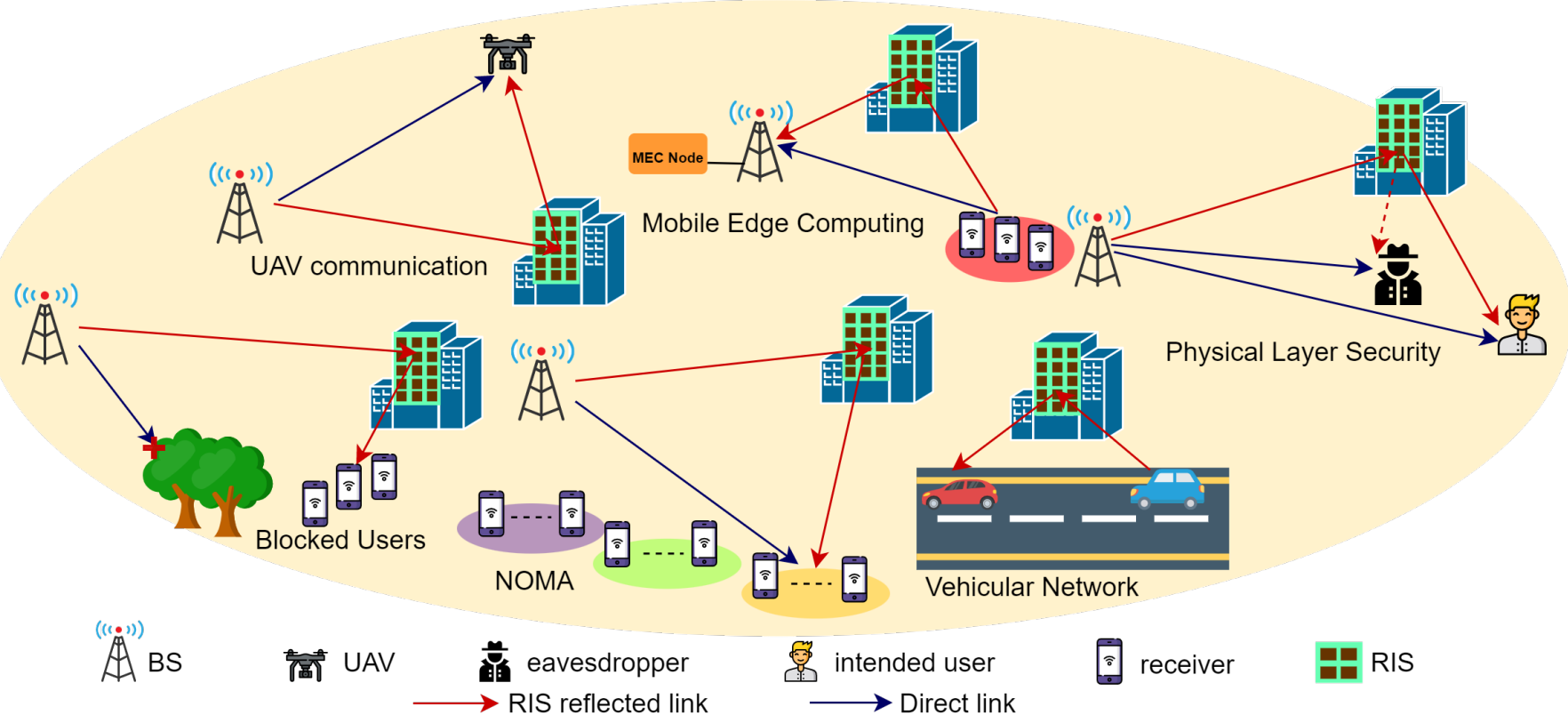

Fig. 1: Emerging applications of RIS for $6 \mathrm{G}$ wireless networks.

and power transfer (SWIPT) networks [29]. In addition, RIS can be leveraged for performance enhancement in vehicularto-everything (V2X) [30], [31], [32], and unmanned aerial vehicle (UAV) networks [33], [34], [35], [36]. Furthermore, RIS can improve the physical layer security (PLS) [37]. The interference mitigation property of RIS can also be applied in device-to-device (D2D) communication to realize massive connectivity. Moreover, RIS can also be integrated with the state-of-the-art 5G technology, massive MIMO, to improve the system performance [38].

Objectives and Contributions. Owing to the abovementioned promising features, the RIS technology has drawn significant attention from the research community. Consequently, a handful of articles, providing the survey and overview of the recent research contributions on various aspects of RIS-assisted communications, have appeared in the literature [39], [40], [41], [42], [9], [12], [37], [43], [44], [45], [46], [47], [48], [49], [50], which are summarized in Table I. Compared to the aforementioned works, this paper is the first effort to systematically and concisely integrate most of the communication and networking aspects of RIS-aided communication; while focusing on the $6 \mathrm{G}$ wireless networks. To this end, the main contributions of this paper can be summarized as follows.

- We overview the fundamentals of RIS technology, including the implementation, hardware architecture, and control mechanisms. We also discuss different deployment strategies of RIS.

- We provide a comprehensive discussion on integrating RISs with emerging communication technologies towards 6G, namely, mmWave and $\mathrm{THz}$ communications, MEC networks, UAV-terrestrial networks, vehicular communication and IoT networks.

- We address the key challenges in designing and implementing the RIS-assisted networks, including the channel state information (CSI) acquisition and passive beamforming optimization.

- Owing to the unrivaled effectiveness of artificial intelligence (AI) based techniques, we review the recent research contributions on AI based algorithms for channel estimation, phase-shift optimization, and resource allocation in RIS-assisted networks.

- To provide useful guidance for further research, we introduce important research directions for realizing RISassisted 6G wireless networks.

The rest of this paper is structured as follows. We first discuss the fundamentals of RIS in Section II. Then, in Section III, we present the emerging use cases of RIS for $6 \mathrm{G}$ communication, followed by the RIS CSI acquisition and passive beamforming design in Section IV. Subsequently, we discuss the benefits of integrating artificial intelligence (AI) and RIS in Section V. Finally, in Section VI, we identify some research opportunities to spark additional research interest, and conclude this paper in Section VII.

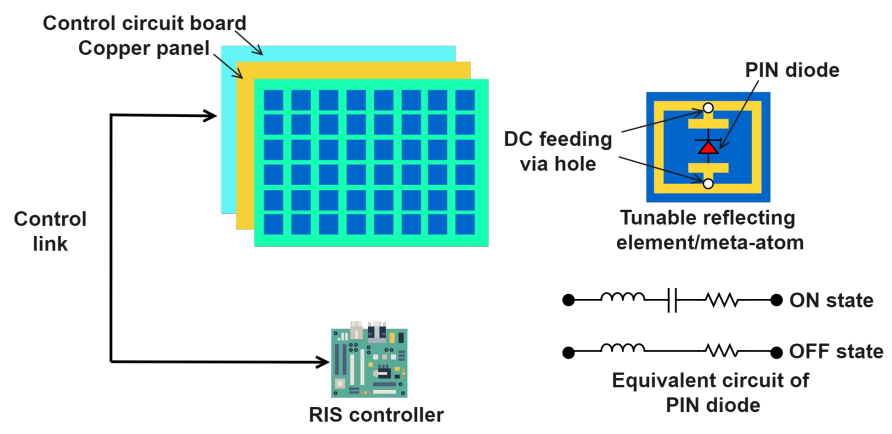

Fig. 2: Hardware architecture of an RIS. 
TABLE I: A list of survey/overview papers on RIS.

\begin{tabular}{|c|c|c|}
\hline Ref. & Year & Major Contributions \\
\hline [39] & 2018 & $\begin{array}{l}\text { Elaborates the key physical properties, working principle and technical details of software controlled meta- } \\
\text { surfaces. }\end{array}$ \\
\hline 40] & 019 & $\begin{array}{l}\text { Immarizes the research studies on RIS, particularly on capacity analyses, spectral optimization, channel } \\
\text { timation, reliability analysis and secure communication. }\end{array}$ \\
\hline [41] & 2020 & $\begin{array}{l}\text { Outlines the different design aspects, analytical approaches and performance metrics for the analysis of } \\
\text { RIS aided wireless networks. }\end{array}$ \\
\hline$[42$ & 220 & $\begin{array}{l}\text { Discusses the working principle, different implementations, and suitable channel models along with the } \\
\text { challenges and opportunities in RIS-assisted wireless communications. }\end{array}$ \\
\hline [9] & م0 & $\begin{array}{l}\text { Provides an overview of RIS applications, state-of-the-art research and future research directions for } \\
\text { realizing RIS-assisted networks. }\end{array}$ \\
\hline [12] & 20 & $\begin{array}{l}\text { jerviews the RIS technology, including the major advantages over existing technologies, hardware } \\
\text { chitecture, signal model, and design challenges for RIS-assisted networks. }\end{array}$ \\
\hline [37] & & Provides an overview of RIS-aided physical layer security for smart and secure wireless networks. \\
\hline TO & 2020 & $\begin{array}{l}\text { Discusses the state-of-the-art research on RIS, including the working principle, various performance } \\
\text { metrics, design aspects, and applications of ML techniques in RIS assisted smart wireless networks. }\end{array}$ \\
\hline [44] & 20 & $\begin{array}{l}\text { Provides an overview of latest deep learning (DL) architectures for RIS-aided wireless networks and } \\
\text { highlight the competitive strengths and limitations of DL-based techniques for RIS-aided networks. }\end{array}$ \\
\hline$[45$ & 020 & $\begin{array}{l}\text { Discusses the crucial challenges for the incorporation of RISs into wireless networks, namely, channel } \\
\text { estimation, passive information transfer, and low-complexity robust system design. }\end{array}$ \\
\hline Fo] & 2020 & $\begin{array}{l}\text { Highlights the key differences and similarities between the RISs and relays, and discusses the potential } \\
\text { applications of RISs in wireless networks operated at millimeter or sub-millimeter frequency bands. }\end{array}$ \\
\hline [47] & 2021 & $\begin{array}{l}\text { Provides an overview of RIS technology in wireless communication, and elaborate its reflection and channel } \\
\text { models, hardware architecture and practical constraints. }\end{array}$ \\
\hline [48] & 2021 & $\begin{array}{l}\text { Discusses the fundamentals, solutions and future aspects of channel estimation in RIS-empowered wireless } \\
\text { communication systems. }\end{array}$ \\
\hline [49] & 202 & $\begin{array}{l}\text { Provides an overview of different RIS models and their consequences for the design of RIS-assisted } \\
\text { wireless communications systems. }\end{array}$ \\
\hline$[30]$ & 2021 & ovides an overview of RIS-assisted BackCom for realizing 6G IoT networks. \\
\hline
\end{tabular}

\section{FUndAMENTALS OF RIS}

In this section, we present the fundamentals, including the RIS's structure and operation, and placement strategies.

\section{A. RIS's Structure and Operation}

The discussion on the implementation, hardware architecture, and control mechanism of RISs is presented as follows.

1) Implementation: The hardware implementation of RIS is based on the concept of programmable meta-surface [12], an artificially developed two-dimensional planner surface consisting of a large number of reflecting elements, called meta-atoms, each of size, smaller than the operating wavelength [51]. By carefully designing the geometrical properties of meta-atoms, i.e., shape, size, arrangement, orientation, etc, the desired signal response of each element can be achieved. However, in wireless communication, the propagation channel is a time varying entity, therefore, a real time channel response for each element is required. To accommodate this, RIS needs to be designed with adjustable reflection coefficients to tune the propagation channel dynamically.
2) Hardware Architecture: As illustrated in Fig. 2, a typical RIS architecture consists of three layers and a controller [12]. A massive number of metallic patches are embedded on a dielectric substrate on the outer layer, that directly interacts with the incident wave. A copper plate is placed behind the outer layer in order to avoid the signal leakage. The inner layer is the control circuit board that communicates with the controller and adjusts the phase-shift and reflection coefficient of each element. The RIS controller, which can be implemented using a micro-controller unit (MCU) or a field-programmable gate array (FPGA) device, acts as a gate way to communicate with the base station (BS) and other network components through a dedicated wired or a wireless link. In a typical scenario, the optimal phase-shifts of RIS, which depends on the CSI, are obtained at the BS station and are communicated to the RIS via controller. The higher clock speed of an FPGA makes it suitable for the wireless communication applications where a faster switching operation is required [52].

3) Control Mechanism: The phase tuning mechanism of RIS depends on the hardware implementation, as summarized 
TABLE II: Summary of different control mechanisms and placement strategies for an RIS

\begin{tabular}{|c|c|c|c|}
\hline Topic & Ref. & Concept & Main Insights \\
\hline \multirow{4}{*}{ Control mechanisms } & [53] & Frequency selective surfaces & $\begin{array}{l}\text { Each element is embedded with a PIN diode which } \\
\text { switches between the two states when DC voltage is varied }\end{array}$ \\
\hline & [54] & Varactor-tuned resonators & $\begin{array}{l}\text { A tunable capacitor is embedded in each element which } \\
\text { realizes the desired phase-shift when biasing voltage is } \\
\text { varied }\end{array}$ \\
\hline & [39] & HyperSurfaces & $\begin{array}{l}\text { The software-controlled surfaces are embedded with elec- } \\
\text { tronic switches to control the electromagnetic response }\end{array}$ \\
\hline & [55] & Liquid crystals & $\begin{array}{l}\text { Each unit cell is loaded with liquid crystal, the dielectric } \\
\text { constant is adjusted by varying the DC voltage, resulting } \\
\text { in desired phase responce }\end{array}$ \\
\hline \multirow{4}{*}{ Placement strategies } & [47] & Centralized terrestrial placement & $\begin{array}{l}\text { All the reflecting elements are grouped as a single RIS } \\
\text { placed close to the BS, termed as BS-side RIS }\end{array}$ \\
\hline & [56] & Distributed terrestrial placement & $\begin{array}{l}\text { The reflecting elements are partitioned into multiple RISs } \\
\text { placed close to users' hot spots, termed as users-side RIS }\end{array}$ \\
\hline & [57] & Hybrid terrestrial placement & $\begin{array}{l}\text { The combination of both the centralized and distributed } \\
\text { placement, where the RIS is placed both at the BS-side } \\
\text { and the users-side }\end{array}$ \\
\hline & [58] & Aerial placement & $\begin{array}{l}\text { RIS is hanged with a UAV or balloon to realize } 360^{\circ} \text { for } \\
\text { angle reflection }\end{array}$ \\
\hline
\end{tabular}

in Table II. In the sequel, we describe the operating principle based on the different realizations proposed for RISs.

Frequency-selective surfaces. In [53], the authors introduced frequency-selective surfaces, where each element is embedded with a positive-intrinsic-negative (PIN) diode. By varying the biasing voltage, the PIN diode can be switched between the two states, i.e., ON and OFF, realizing a phase-shift of $\pi$ in radians. The PIN diode based implementation of RIS elements, is also illustrated in Fig. 2. In general, to realize $K$ phase-shift levels, $\log _{2} K$ diodes are required. However, it is challenging to implement such high precision design because of the limited size of RIS elements and greater number of pins required to control each element. Therefore, it is feasible to implement only discrete phase-shifts [12].

Varactor-tuned resonators. Another approach for realizing the RIS phase configuration is through varactor-tuned resonators [54]. The basic idea of this implementation is to change the resonant frequency of each element, which can be achieved by embedding a tunable capacitor in each element, thus the desired phase-shift is realized by varying the biasing voltage across the capacitor.

HyperSurfaces. The working mechanism of HyperSurfaces is based on the concept of coating objects with the electromagnetic materials to control the behaviour of radio waves in a software controlled fashion [39]. These software-controlled surfaces are embedded with electronic switches to dynamically control the response of the surface towards the impinging signals. Moreover, a HyperSurface can realize various phenomenon such as wave steering, wave polarization, wave absorption, depending upon the programming of the software. Liquid-crystal based reflectors. The real time reconfiguration of RIS elements can be achieved through functional materials, such as liquid crystals and graphene. In [55], the authors proposed a liquid-crystal based design, where the reconfigurability is achieved by varying the direct current voltage across the liquid crystal filled elements, as a result the dielectric constant of each element can be adjusted in real time.

In particular, electronic devices have been widely adopted in practical implementation due to the fast response time, low reflection loss, low hardware cost and energy consumption [9]. For instance, according to [62], the switching time for PIN didoe is approximately 0.2 microsecond $(\mu \mathrm{s})$, which is much less than the coherence time of the wireless channel. Consequentially, electronic devices are most suited for phase reconfiguration of RIS in wireless applications. The readers interested in having detailed information on various implementations of RISs are referred to the paper [41].

\section{B. Deployment}

In order to achieve optimum performance, RIS must be carefully deployed in the wireless network. Since RIS is lightweight and of conformal geometry, it can be easily deployed on buildings facades, walls, and ceilings. For terrestrial RIS, there are two popular strategies for deployment: i) centralized deployment at the BS-side [47], and ii) distributed deployment at the user-side [56]. Both the strategies aim at minimizing the product distance path loss, associated with the BS-RIS and RIS-users' links [47] and have different pros and cons. BS-side RIS outperforms the user-side in terms of passive beamforming gain because each user can be served by all reflecting elements. This is not the case with distributed user-side deployment where each user is served with only 
TABLE III: Summary of Emerging Applications of RIS. "DL" and "UL" represent downlink and uplink, respectively, and MISO and SISO represent multiple-input single-output and single-input single-output, respectively.

\begin{tabular}{|c|c|c|c|c|c|c|c|}
\hline Topic & Ref. & Scenarios & Direction & Users & RISs & Performance metrics & Techniques \\
\hline \multirow{3}{*}{ mmWaves } & [18] & SISO & $\mathrm{DL}$ & Single user & Single RIS & Outage probability & In-lab experiments and analytical modelling \\
\hline & [19] & MISO & DL & Single user & Multiple RISs & Received signal power & Gradient projection method \\
\hline & [20] & MIMO & DL & Single user & Single RIS & Channel capacity & Analytical modelling \\
\hline \multirow{3}{*}{ THz } & {$[21]$} & SISO & $\mathrm{DL}$ & Multiple users & Single RIS & Sum rate & BCS algorithm \\
\hline & [22] & MISO & $\mathrm{DL}$ & Multiple users & Single RIS & Energy efficiency & CMA-ES and Dinkelbach's method \\
\hline & {$[23]$} & SIMO & UL & Multiple users & Single RIS & Computational latency & BCD algorithm \\
\hline \multirow[t]{5}{*}{ MEC } & [25] & SISO & UL & Multiple users & Single RIS & Computational resources & SCA and Lagrange duality method \\
\hline & {$[24]$} & MIMO & UL & Multiple users & Single RIS & Sum computational bits & Lagrange duality and sub-gradient method \\
\hline & [26] & SIMO & UL & Multiple users & Single RIS & Computational resources & BCD algorithm \\
\hline & [28] & MISO & DL & Multiple users & Single RIS & Power consumption & CVX and DC Techniques \\
\hline & [27] & SIMO & UL & Multiple users & Single RIS & SINR and learning errors & SCA, AO and ADMM based algorithms \\
\hline \multirow{4}{*}{ UAVs } & {$[33]$} & MISO & $\mathrm{DL}$ & Multiple users & Single RIS & Transmision capacity & Q-learning and neural networks \\
\hline & [34] & MISO & DL & Single user & Multiple RISs & Received power level & SCA method \\
\hline & [35] & SISO & DL & Single user & Single RIS & Average achievable rate & SCA method \\
\hline & [36] & SISO & DL & Single user & Single RIS & Received signal strength & 3GPP ground-to-air channel models \\
\hline \multirow{3}{*}{ VANETs } & {$[30]$} & SISO & DL & Mutiple users & Single RIS & Sum V2I capacity & RAIVC algorithm \\
\hline & [31] & MISO & DL & Single user & Multiple RISs & Received power level & Optimum RIS positioning algorithm \\
\hline & [32] & MIMO & UL & Multiple users & Single RIS & Collosion Probability & CSMA/CA \\
\hline \multirow{4}{*}{ IoTs } & {$[29]$} & MIMO & DL & Multiple users & Single RIS & Weighted sum rate & BCD algorithm \\
\hline & [59] & MISO & DL & Single user & Single RIS & Spectral efficiency & Power splitting optimization \\
\hline & [60] & SISO & UL & Multiple users & Multiple RISs & Sum rate & Stackelberg game \\
\hline & [61] & SISO & DL & Single user & Single RIS & Average capacity & Asymptomatic derivation \\
\hline
\end{tabular}

small portion of total RIS elements. Moreover, as compared to the user-side, BS-side RIS offers large network coverage and low signalling overhead. This deployment, however, suffers from the unavailability of line-of-sight (LoS) links for all users in the network.

Recently, [57] proposed a hybrid RIS deployment strategy by combining the benefits of both the BS-side and user-side deployment strategies. The results demonstrate the superiority of the hybrid RIS deployment strategy over the BS-side and user-side strategies in terms of minimum achievable rate. However, hybrid strategy poses various challenges, from user association and channel acquisition to placement and surface partitioning. Despite the aforementioned benefits of hybrid deployment strategy, the terrestrial RIS suffers from $180^{\circ}$ halfspace reflection which limits the coverage range. Fortunately, this issue can be resolved by an aerial RIS, carried by a balloon or a UAV, which can realize $360^{\circ}$ full-space reflections [58], [63]. A summary of different deployment strategies for RIS is presented in Table II.

\section{EMERging APPLICATIONS OF RIS}

Recent research studies have revealed the capability of RIS to improve the spectral efficiency and coverage range of the wireless networks in a cost-effective and energy-efficient manner. In this section, we present the important aspects of integrating RIS with other emerging communication technologies as mmWave and THz communications, MEC networks, VANETs, UAVs, and IoT networks. The existing works on the applications of RIS in wireless networks are summarized in Table III.

\section{A. RIS-assisted mmWave and $\mathrm{THz}$ Communications}

Due to the availability of immense spare bandwidth in highfrequency spectrum, mmWave and $\mathrm{THz}$ communications are promising solutions for rate-hungry wireless applications such as virtual/augmented reality and online gaming, etc. However, the vulnerability of millimeter and THz waves to signal blockage, atmospheric attenuation, molecular absorption, and severe path loss limits the operational range and communication reliability. Although, the performance can be improved by leveraging an increasing number of BS antennas, however, this incurs high hardware costs and energy consumption. Therefore, to address these limitations, RIS can be applied to mmWave and $\mathrm{THz}$ communications to enable effective additional paths to establish communication when direct BS to users' links are blocked, as illustrated in Fig. 3. Moreover, the RIS passive beamforming gain can enhance the communication performance in case of poor propagation conditions, hence enabling reliable and energy-efficient communication in a cost-effective fashion.

Recently, in [18], the authors investigated the performance of $60 \mathrm{GHz}$ reconfigurable surface, consisting of 224 reflecting elements, for the indoor mobile mmWave communications, where the direct links are assumed to be blocked. The authors demonstrated the in-lab test bed based experimental results for the proposed model which validated the beam steering performance of the proposed design. Furthermore, the authors derived the outage probability expressions for the proposed design and minimized the outage probability for the optimal location of RIS. In addition, in [19], the authors considered the channel capacity optimization for the RIS-assisted indoor mmWave communication and proposed low-complexity algo- 


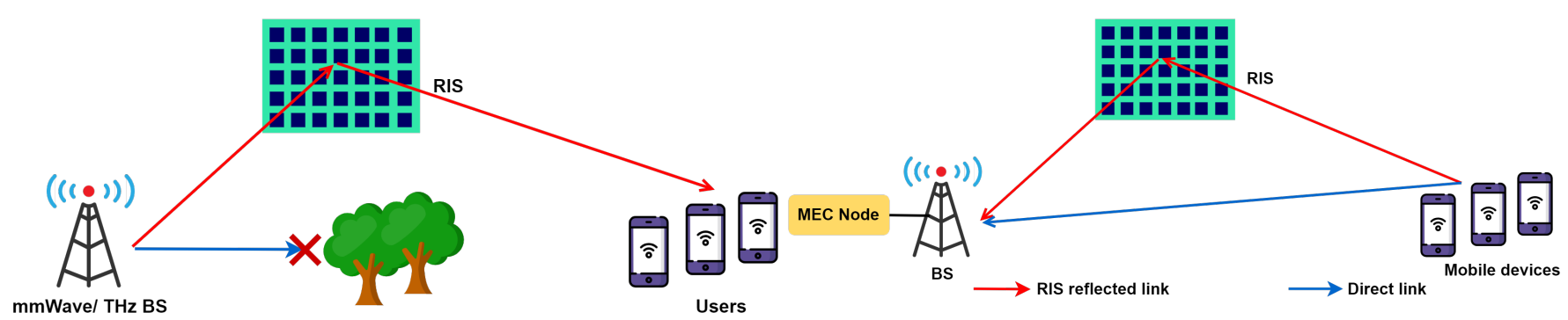

Fig. 5: An illustration of RIS-assisted MEC system.

Fig. 3: An illustration of RIS-assisted mmWave/THz communication system.

rithms for the optimization of RIS phase-shifts and transmit phase precoder. Moreover, in [20], the authors considered the joint optimization of transmit beamforming and RIS phaseshifts for received signal power maximization problem for both the single and multi RIS-assisted systems. The results, as illustrated in Fig. 4, demonstrate the significant performance improvement in terms of average received SNR with the aid of RIS. Moreover, the results presented in [20] provide useful insights into the performance loss caused by the discrete phase-shift design.

To unveil the potentials of RISs for THz communication, in [21], the authors considered the joint optimization of RIS position, RIS phase-shifts, sub-channel allocation, and power control to maximize the sum rate of the RIS-assisted $\mathrm{THz}$ communication system. In order to solve the non-convex problem for the generalized number of users, the authors proposed a a block coordinate searching (BCS) based algorithm. The results clearly depict the superiority of the proposed approach over the benchmark schemes. Furthermore, in [22], the authors formulated an energy efficiency maximization problem for the RIS-assisted THz communication system and employed the covariance matrix adaptation evolution strategy (CMA-

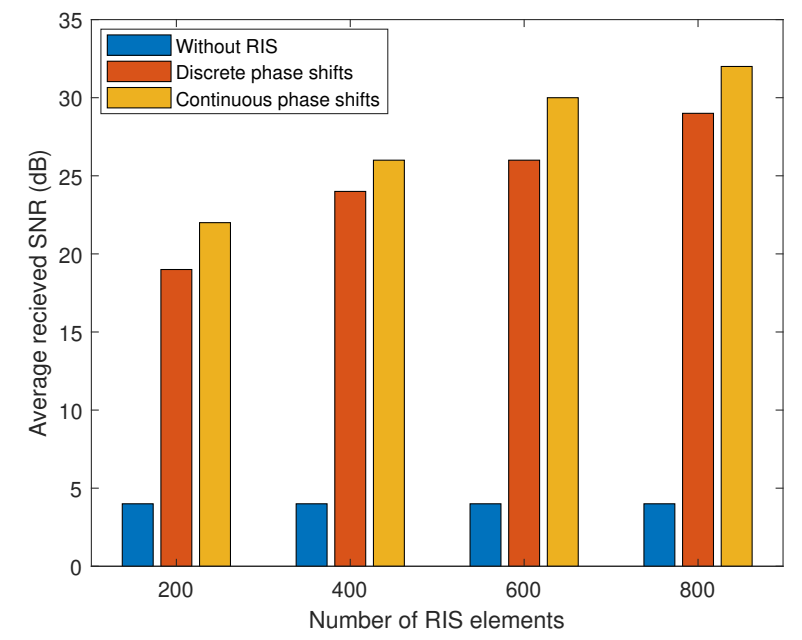

Fig. 4: Average received SNR of an RIS-assisted mmWave communication system versus the number of RIS elements [20].
ES) and Dinkelbach's method based algorithm to optimize the transmit beamforming, RIS phase-shifts, and power allocation.

\section{B. RIS-assisted MEC networks}

The future IoT network has the potential to realize diverse applications such as automated home appliances, smart cities, autonomous driving, smart health monitoring systems, intelligent transportation, industrial automation, and emergency management [64]. However, the massive deployment of lowpower wireless devices increases the load on the network; since these devices are incapable of performing computationalintensive tasks and thus are unable to support most of the resource-intensive applications. Fortunately, in this regard, MEC is a viable solution in terms of computational capabilities and resource management. MEC servers share the burden of wireless network devices by performing partial computation at the edge of network nodes, i.e., access points (APs), thus mitigating the computational latency in real-time networks.

Despite the potentials of the MEC network, it is challenging to fully utilize its capabilities in practical scenarios, where the network is highly dynamic with the communication links changing in fractions of time. Moreover, when the mobile devices are far away from the MEC node, the severe path loss leads to excessive offloading delays, which limits the performance of the MEC network. Nevertheless, as illustrated in Fig. 5, RIS can be integrated into the MEC network to address these challenges. Thus, the RIS passive beamforming

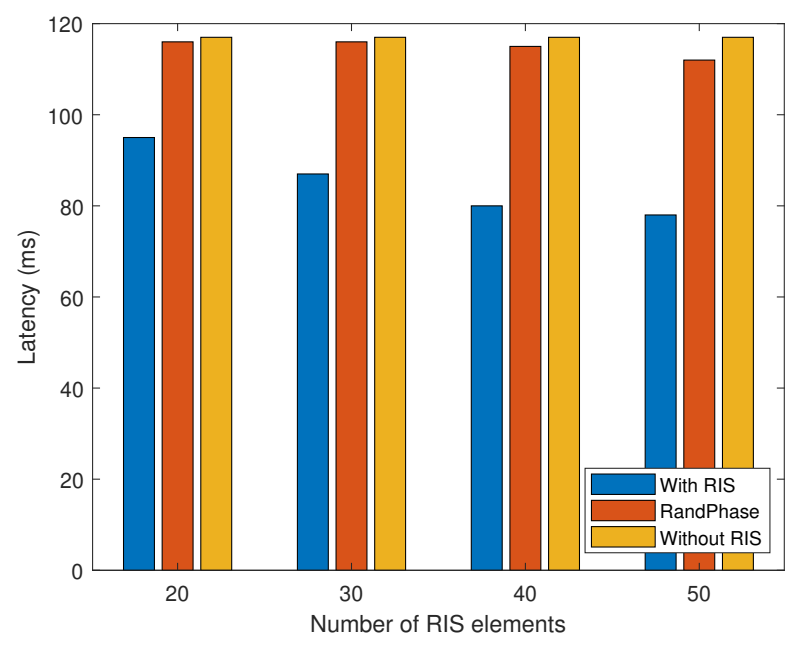

Fig. 6: Latency versus the number of RIS elements [23]. 


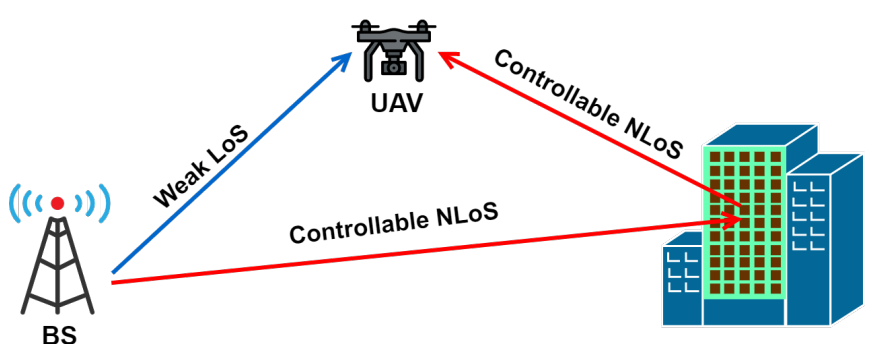

Fig. 7: System model for RIS-assisted cellular UAV communications [36].

gain can significantly improve the wireless channel capacity, enabling the mobile devices to offload the computationintensive tasks to the edge nodes without incurring high energy consumption. Furthermore, in a large-scale MEC network with an increasing number of edge nodes and mobile devices, RIS can be exploited to offload the computation tasks to the distant nodes. Hence, the total load gets distributed over the entire network, which results in better resource utilization and latency minimization.

Recently, the RIS-assisted MEC networks have attracted wide research attention. For instance, the authors in [23], [24] demonstrated that the RIS-assisted MEC network outperforms the conventional MEC network in terms of computational offloading, as illustrated in Fig. 6 [23]. The authors utilized the block coordinate descent (BCD) algorithm and successive convex approximation (SCA) method in [23] and [24], respectively, to solve the latency minimization problem. Moreover, in [25], the authors considered an RIS-assisted wireless-powered (WP) MEC system, where the RIS is employed to reduce the total energy consumption. The results in [25] demonstrate the significant reduction in energy consumption with the aid of RIS. In [26], the authors extended the work presented in [23] by considering the network cost and rate as performance metrics. Moreover, in [28], the authors exploited the CVX and difference of convex (DC) functions to jointly optimize the downlink transmit power and resource allocation strategies for RIS-assisted MEC network. To reduce the computational complexity, the alternating optimization (AO) framework based on SCA and alternating direction method of multiplier (ADMM) is proposed in [27] to optimize the transmit power and RIS phase-shifts.

\section{RIS-assisted UAV networks}

UAVs, commonly known as drones, equipped with the advance transmitters, receivers and batteries, can be utilized for relaying, gathering of data, and for secure transmission of information [65]. Due to high mobility and flexible nature of this technology, it can be deployed as an aerial platform for communication purposes in the existing wireless technologies (terrestrial) such as cellular networks [66].

The UAV-enabled wireless communication can be improved by incorporating RISs. For instance, in a dense urban environment or in mmWave communications, the line-of-sight (LoS) link between the UAV and the ground node may be blocked, which degrades the communication performance. In such a

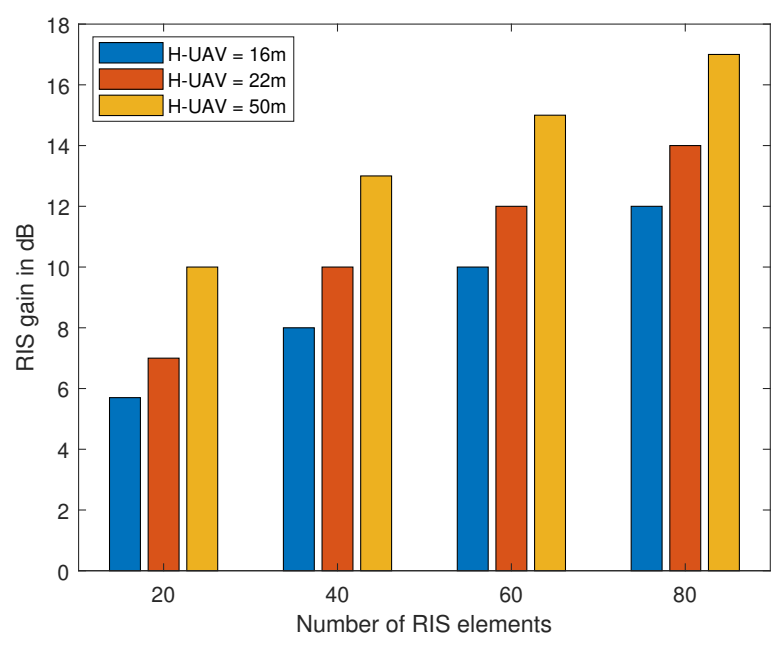

Fig. 8: RIS gain vs. number of RIS elements for different UAV heights [36].

scenario, RIS can be applied to improve the communication performance by enabling effective communication links. In addition, the ability of RIS to destructively combine the signals can be utilized to mitigate the inter-cell interference problem in future UAV-enabled networks, which arises due to the favourable channel conditions between the terrestrial BSs and the UAVs in the adjacent cells. Moreover, in UAV-assisted relay networks, the aerial RIS can significantly improve the coverage and connectivity of the network as compared to terrestrial RIS [33]. The cellular communication of the UAVs, which suffers from the down-titled antennas of the BSs, can be enhanced via controllable reflection from RIS, as shown in Fig. 7. The direct and RIS reflected links are combined coherently at the UAV, resulting in improvement in overall signal strength at the UAV.

Recently, in [34], [35], the authors considered the joint optimization of passive beamforming and trajectory of UAVs to maximize the achievable rate of the RIS-assisted UAV communication system. The results in [34], [35] demonstrate the substantial improvement in the communication performance with the aid of RIS. Moreover, RIS can be deployed to improve the cellular communication of the UAVs, which usually suffers from weak signal strength due to the down tilted BS antennas that are optimized to serve the ground users. In this regard, in [36], the authors optimized the RIS phase-shifts to direct the reflected signal towards the specific UAV in order to maximize the received signal power. As illustrated in Fig. 8, the results in [36] demonstrate the increase in RIS channel gain with the increase in number of reflecting elements. Moreover, the maximum RIS gain can be achieved when a UAV is closer to the ground. In [33], the authors considered an intelligent reflector mounted on a UAV, which can establish multiple LoS communication links to mitigate the blockage.

\section{RIS-assisted VANETs}

The intelligent transport systems (ITS) and autonomous driving have been recognized as cutting-edge technological 


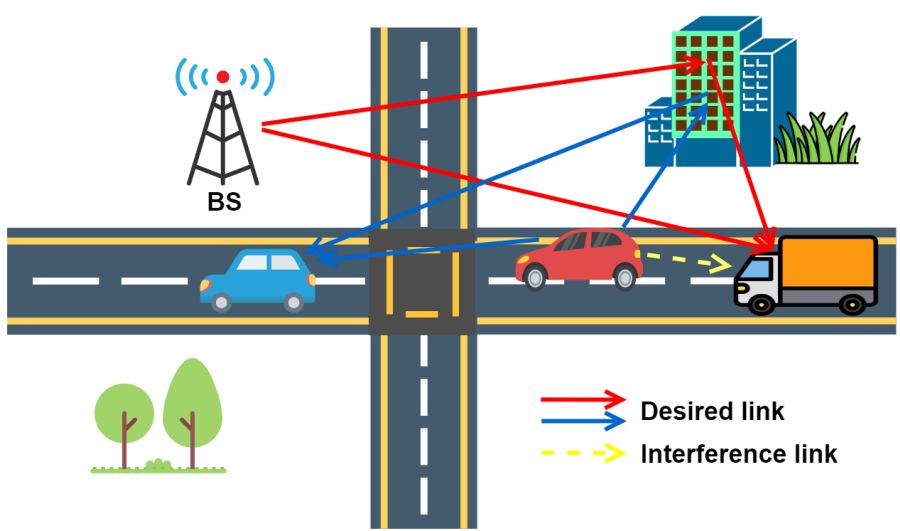

Fig. 9: An illustration of RIS-assisted vehicular communication network.

paradigms for $6 \mathrm{G}$ wireless networks. In this context, V2X is a promising solution to enable better road safety, fuel savings, traffic efficiency, and better road management for the daily travel. However, due to the dynamic nature of the wireless propagation environment, especially considering the intermittent connectivity due to fast moving vehicles, the transmission links in vehicular communication are highly unstable. In order to address the above mentioned challenge, RIS can be applied to vehicular communication, as illustrated in Fig. 9, to improve the channel conditions via intelligent signal reflections, thereby improving the communication performance and coverage range. However, to fully exploit the potentials of RISs in V2X, the RIS phase-shifts need to be jointly optimized with the resource management and vehicular scheduling.

Recently, in [30], the authors proposed an iterative method called resource allocation algorithm for RIS-assisted vehicular communication (RAIVC) to solve the formulated resource allocation optimization problem. Fig. 10 demonstrates that the proposed RAIVC algorithm outperforms the benchmark

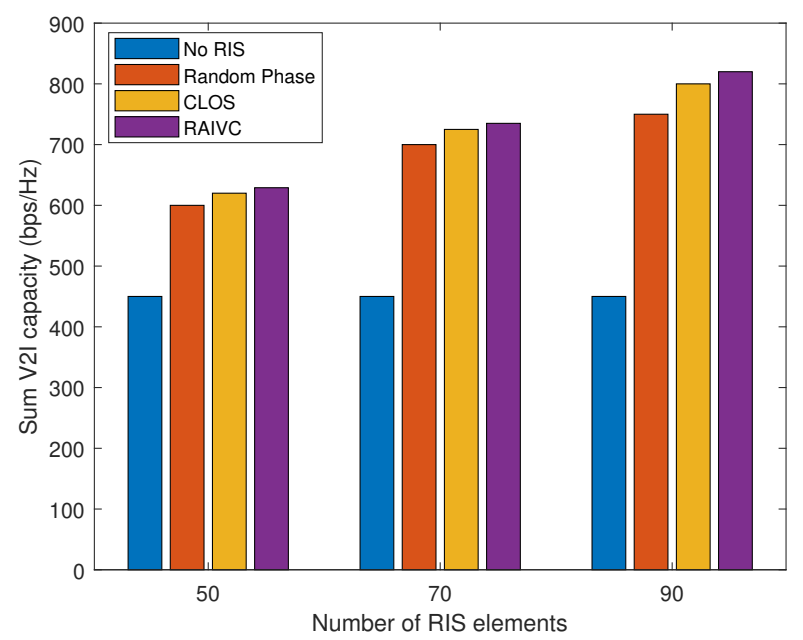

Fig. 10: Sum V2I capacity versus the number of RIS elements [30].

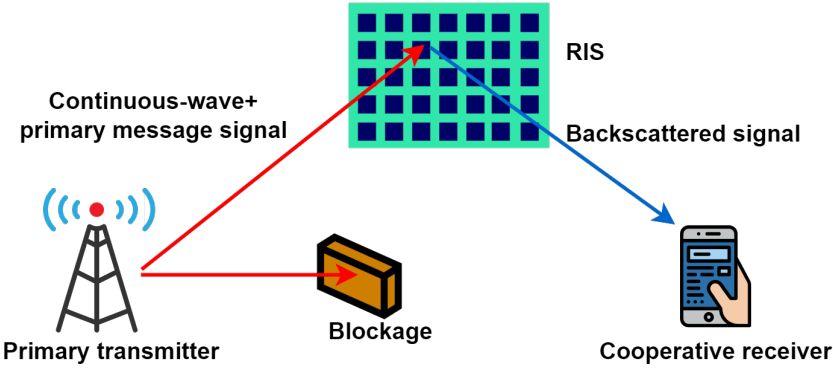

Fig. 11: An illustration of RIS-assisted backscatter communication system.

approaches.

In vehicular communication, the obstacles that affect the propagation of transmitted signal may be physically fixed or moving with respect to the source vehicle. Traditional wireless communication system experiences small-scale fading due to the presence of these obstacles. RIS is considered as a suitable approach to mitigate these issues pertaining to vehicular communication [30], [67]. RISs have the potential to enhance coverage area and capacity of VANETs in $6 \mathrm{G}$ wireless communications [32], [39]. In V2X communications, links are highly dynamic which makes the system unreliable in terms of variable SNR and fading effect. RIS provides reliability to V2X systems by software-controlled configuration of reflecting surfaces. This ability to reconfigure reflecting surfaces in RISs gives additional control over reflection angle and phase-shifts which consequently enhances received signal strength as well as eliminates channel fading [31]. According to the authors in [32], a carrier sense multiple access/collision avoidance (CSMA/CA) technique may be used for packet loss minimization by leveraging RISs to enhance performance, which is applicable towards crucial information transfer in use cases like cooperative driving or pedestrian detection. Although the research conducted in the field of RIS assisted

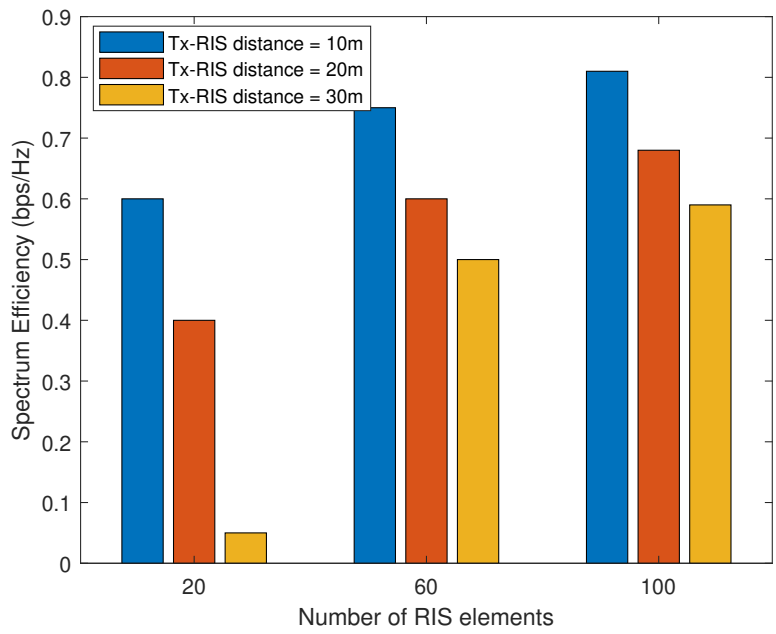

Fig. 12: Spectrum efficiency of an RIS-assisted backscatter communication system versus the number of reflecting elements [59]. 
vehicular networks is still in infancy, it clearly elaborates the significance of RIS in ITS, since these reconfigurable surfaces have the potential to resolve most of the issues in vehicular communication.

\section{E. RIS-assisted IoT networks}

An IoT network connects massive number of devices to communicate and exchange information over the internet enabling diverse applications such as smart homes, health care, industrial automation, and many more. Despite the rapid advancements in IoT domain, there are some major challenges that need to be addressed to unleash the full potential of IoT networks. Firstly, the massive low-power devices in IoT networks suffer from short operational range, therefore, efficient powering of densely deployed IoT devices is required. Secondly, the scarcity of the radio spectrum limits the performance of IoT network. In these contexts, backscatter communication and SWIPT have emerged to address the energy limitations of IoT devices, and cognitive radio (CR) to address the spectrum scarcity issue. There has been considerable research effort lately for investigating these issues. However, these technologies are insufficient own their own to meet the demands of 6G IoT networks. Therefore, to meet the stringent demands on energy harvesting, data reliability, throughput, energy and spectrum efficiency, and operational range, the RIS ability to coherently combine the signals to scale the received signal strength can be utilized to improve the system performance.

Inspired from the benefits of RISs, recently, the research community investigated the integration of RISs in backscatter communication [50], [59], SWIPT [81], [29], and cognitive radios [60], for realizing 6G IoT networks. Specifically, in [29], the authors explored the RIS-assisted SWIPT systems for enhancing the energy harvesting performance of energy receivers (ERs). A weighted sum-rate (WSR) maximization problem was formulated for joint optimization of transmit precoding (TCP) matrices of the BS and passive beamforming matrices of the RIS, under unit modulus constraint on the phase-shifts, by using block coordinate descent (BCD) algorithm.

Furthermore, in [59], the authors integrate the ambient backscatter communication with RIS by replacing the antennatags in backscatter by RIS elements to address the fundamental short operation range in traditional backscatter communication, as shown in Fig. 11. The authors employed the RIS for encoding the secondary information onto the RIS phaseshifts, while relaying the primary information data. The authors demonstrated that the RIS-assisted backscatter system achieves high data rate than the conventional backscatter system without RIS. Furthermore, as shown in Fig. 12, the RIS-assisted backscatter system achieves high spectrum efficiency for smaller distance between the transmitter and RIS. Furthermore, the authors in [82] experimentally demonstrated the improvement in the performance of backscatter communications with the aid of RIS.

The authors in [60] investigated a CR IoT system with multiple RISs. The RISs assist the secondary IoT devices to improve the spectral efficiency by sharing spectrum with the primary access points. In the considered far-field path loss model, each RIS element acts as a scattering object and directs the signal from the IoT device in a particular direction to achieve maximum signal gain. The results indicate better system performance with the aid of RIS. In [61], the authors analyzed the IoT network with RIS-enabled access points over generalized fading and shadowing. The authors demonstrated the benefits of employing RIS-eabled access points in terms of bit error rate (BER), outage probability (OP) and average capacity. Furthermore, the authors highlighted the importance of balance between the cost and number of deployed RIS elements in RIS-enabled IoT networks.

\section{RIS ChANNEl ACQUiSition AND PASSIVE BEAMFORMING DESIGN}

Besides the promising advantages, in practice, RIS brings new challenges, including the CSI acquisition and passive beamforming optimization. To this end, we address the key challenges in designing and implementing the RIS-assisted networks. A summary of existing works on RIS channel acquisition and passive beamforming optimization is listed in Table IV.

\section{A. Channel Acquisition}

The performance gains brought by RIS highly rely on the availability of CSI, which is quite challenging to acquire in practice. By turning off the RIS, the direct channel can be estimated using conventional channel estimation techniques. However, it is infeasible to estimate the transmitter-to-RIS and RIS-to-receiver channels since the passive RIS elements lack signal processing and transmission capabilities. Therefore, most of the existing channel estimation schemes directly estimate the cascaded channel, i.e., the product of transmitterto-RIS and RIS-to-receiver channel, which is, fortunately, sufficient in most applications. In the sequel, we present different CSI acquisition approaches for RIS-assisted systems. ON/OFF-Based Channel Acquisition. One practical approach for RIS's cascaded CSI acquisition is by utilizing the element-by-element ON/OFF-based channel estimation scheme, in which one scattering element is active at a time [68]. However, for large RISs with massive number of elements, this scheme incurs high training overhead. Moreover, the ON/OFF-based approach suffers from severe power loss since only element is active at a time and consequently the reflected signal is very weak, which degrades the channel estimation accuracy. To reduce the training and improve the channel estimation accuracy, in [83], the authors proposed an element grouping based channel acquisition scheme, where the the total number of RIS elements are divided into subsurfaces, hence, only the cascaded channel associated with the sub-surface needs to be estimated.

Discrete Fourier Transform (DFT)-Based Channel Acquisition. To further improve the channel estimation accuracy and compensate for the power loss, the all-ON RIS reflection pattern can be employed with discrete Fourier Transform (DFT) protocol [70]. In the proposed protocol, the entire cascaded channel estimation phase is divided into multiple sub-phases. In each phase, the optimal RIS configuration is 
TABLE IV: Summary of representative works on RIS channel acquisition and passive beamforming optimization.

\begin{tabular}{lll}
\hline & & \multicolumn{1}{c}{ RIS Channel acquisition } \\
\hline Ref. & System setup & Main contributions \\
\hline$[68]$ & Single-user, narrow-band & ON/OFF-based channel acquisition for the RIS-assisted SISO system \\
\hline$[69]$ & Single-user, broad-band & ON/OFF-based channel acquisition with RIS element grouping for the RIS-assisted SISO system \\
\hline$[70]$ & Multi user, narrow-band & DFT-based channel acquisition for the RIS-assisted MISO system \\
\hline$[71]$ & Multi-user, narrow band & Anchor-assisted channel estimation approach for RIS-aided MISO system \\
\hline$[72]$ & Single-user, narrow-band & Compressed-sensing-based channel estimation utilizing the sparsity of the channels in the angular domain \\
\hline$[73]$ & Multi-user, narrow-band & Compressed-sensing-based channel estimation utilizing the sparsity of the channels in the angular domain \\
\hline & & \multicolumn{1}{c}{ RIS passive beamforming optimization } \\
\hline Ref. & System setup & Design objective and optimization techniques \\
\hline$[74]$ & Multi-user MISO & Power minimization, AO and SDR approach \\
\hline$[75]$ & Multi-user MISO & Power minimization, branch-and-bound and successive refinement algorithm \\
\hline$[76]$ & Single-user OFDM & Rate maximization, AO and duality based algorithm \\
\hline$[77]$ & Multi-user MIMO & Weighted sum-rate maximization, AO, SCA and fractional programming \\
\hline$[78]$ & Multi-user MISO & Minimum SINR maximization, Penalty method and ADMM approach \\
\hline$[79]$ & Multi-user MISO & Energy efficiency maximization, AO, gradient descent and SFP optimization method \\
\hline$[80]$ & Multi-user MISO & Energy efficiency maximization, AO and SCA method \\
\hline
\end{tabular}

specially designed as one of the columns of the DFT matrix. The overall reflection matrix at the end of the multiple subphases has been proved to be the optimal choice to ensure the channel estimation accuracy. Although the DFT protocol improves the SNR, however, similar to ON-off-based scheme, the required channel estimation overhead is still high.

Anchor-Based Channel Acquisition. The channel estimation schemes for RIS-assisted single-user system incur high training overhead for a multi-user counterpart since the required pilot overhead is the product of the number of users and RIS elements, which is practically infeasible. In order to tackle this challenge, in [71], the authors proposed a new anchorassisted channel estimation approach, where two anchor nodes, i.e., N1 and N2, deployed close to RIS, assist in estimating the cascaded channel, which significantly reduces the training overhead. Based on the pilot symbols transmitted by the two anchor nodes, the cascaded channel between the anchor nodes and BS, i.e., N1-RIS-BS and N2-RIS-BS channels, and the RIS reflected channel between the two anchor nodes, i.e, N1RIS-N2, are estimated. The BS-RIS channel is then calculated based on the estimated N1-RIS-BS, N2-RIS-BS, and N1-RISN2 channels.

Sparsity-Based Channel Acquisition. For the channel estimation of RIS-assisted single user MISO/MIMO systems, the required pilot overhead scales up the number of antennas at the BS or the user, which is prohibitive in practice. Therefore, to reduce the training overhead and for efficient decomposition of cascaded channel, special properties of the RIS associated channels, such as the sparsity, can be utilized. In this context, in [72], the authors proposed a channel estimation scheme for RIS-assisted mmWave system by exploiting the inherent sparsity of the mmWave channels in the angular domain and utilizing the properties of KatriRao and Kronecker products. In addition, in [73], the authors studied the channel estimation problem for RIS-assisted multi-user MIMO system by exploiting the sparsity of cascaded channels and compressive sensing technique. However, the required pilot overhead is still high for these sparsity-based angular channel estimation schemes, since in the angular domain, the sparsity of the cascaded channel is comparatively less significant than the channels in a conventional system without an RIS.

Codebook-Based Channel Acquisition. The aforementioned channel estimation techniques incur high training overhead in the case of large RISs. In this regard, the codebook-based approach is a promising and scalable solution to attain the pre-designed set of RIS phase-shifts. However, the channel estimation overhead scales up with the size of the codebook. Hence, for efficient communication, it is imperative to design small RIS codebooks capable of achieving high performance gains. Motivated by the benefits of small codebooks, the authors in [84] proposed a quadratic phase-shift design and presented an analytical framework for the RIS phases-shits in terms of the codebook size. Moreover, the authors analyzed the trade-off between the power efficiency and the codebook size. The simulation results reveal the effectiveness of the proposed quadratic phase-shift design over the linear phase-shifts.

The above mentioned channel estimation schemes are based on an RIS with all passive elements. In the sequel, we present active-channel-sensor-based channel estimation scheme.

Active-Channel-Sensor-Based Channel Acquisition. In this approach, each RIS elements is equipped with low-power sensors for sensing channel information. Hence, this semipassive configuration of RIS generally operates in two modes: (i) channel sensing mode, where all the reflecting elements are turned off and the sensors are activated to receive the pilot symbols from the BS/users in order to estimate the uplink/downlink channel to the RIS; (ii) reflecting mode, where the sensors are deactivated and reflecting elements are reconfigured according to the channel estimated in the channel sensing mode to intelligently reflect the incident signals. Different from the semi-passive configuration, the full passive RIS can only estimate the cascaded user-RISBS channel. Although the semi-passive configuration enables the environment learning capabilities, however, equipping the RIS with sensing elements increases the cost and power consumption [9]. Moreover, there is a paucity of research contributions to design the channel estimation protocols for semi-passive RIS, therefore the performance and cost of this 
approach as compared to fully passive RIS is unclear at this stage.

\section{B. Passive Beamforming Optimization}

The most attractive aspect of RIS is its ability to intelligently reflect the impinging signal in the desired direction. Hence, RIS phase-shifts need to be appropriately designed in order to fully reap its potentials. In the sequel, we review the main optimization formulations and solutions proposed for RISassisted wireless networks.

1) Optimization Use cases: The typical design objectives include SNR or rate maximization, transmit power minimization, and EE performance maximization, as described below.

Transmit Power Minimization. The authors in [74], formulated and solved the transmit power minimization problem under users' individual SINR constraints through the joint optimization of transmit beamforming at the BS and reflect beamforming at the RIS. To tackle the non-convex optimization problem, the authors exploited the SDR technique to obtain the high quality sub-optimal solution. However, the computational complexity of SDR is very high for largescale RISs, therefore, to reduce the computational overhead, the authors devised an alternating optimization based efficient algorithm to optimize the transmit beamforming and RIS phase-shifts in an iterative manner. Considering the hardware limitation of RIS phase-shifts, the authors in [75] study the power minimization problem for RIS-assisted multi-user MISO system, where each RIS element can realize only a finite number of phase-shifts. The authors proposed a lowcomplexity successive refinement based algorithm, where the optimal discrete phase-shifts of each element is determined in an iterative manner while keeping the phase-shifts of other elements fixed. The numerical results revealed that the RIS with 1-bit phase sifters can achieve the same asymptotic performance as in the case with continuous phase-shifts.

SNR or Rate Maximization.The authors in [76] discussed an RIS-assisted orthogonal frequency division multiplexing (OFDM) system, where an RIS is deployed to assist the downlink information transmission. A joint optimization problem is considered to maximize the achievable rate by jointly optimizing the BS transmit power allocation and the RIS phase-shifts. Based on the alternating optimization framework, the authors proposed a high-quality sub-optimal solution to optimize the power allocation and RIS phase-shifts through SCA and SDR techniques, respectively. Furthermore, the authors in [77] investigated the RIS-assisted multi-user downlink MISO system while considering practical discrete phase-shift constraints for the RIS. The joint optimization of active beamforming at the BS and passive beamforming at the RIS is considered. Owing to the non-convex nature of the optimization problem, the authors employed the Lagrangian dual transformation to decompose the problem and alternatively optimize the active and passive beamforming. The simulation results reveal the superiority of the proposed algorithm over the baseline schemes. Regarding to the SINR maximization problem, the authors in [78] optimized the beamforming design to maximize the SINR in a downlink RIS-assisted multi-user MISO system.

EE Maximization. Besides the power minimization and rate or SINR maximization, the RIS-aided wireless networks can also help to improve the overall EE performance. Focusing on an RIS-assisted multi-user MISO downlink scenario, the authors in [79] optimized the transmit power allocation and RIS phase-shifts to maximize the system EE. The simulation results illustrate that the proposed algorithm, capitalizing on alternating maximization, gradient descent search, and sequential fractional programming, achieves $300 \%$ higher EE as compared to conventional multi-antenna relaying. Furthermore, the authors in [80] considered a EE maximization problem for an RIS-assisted MISO system with rate splitting multiple access (RSMA). The authors exploited the SCA technique to iteratively optimize the RIS phase-shifts and BS transmit beamforming. The simulation results reveal that the proposed approach achieve better EE as compared to the conventional OFDMA and NOMA schemes.

2) Codebook-Based Optimization: The optimization of RISs with a massive number of elements impose a crucial challenge for transmission in an online mode. To cope with the aforementioned challenge, the codebook-based approach is a promising and scalable solution for the phase-shift optimization of large RISs. In this context, the authors in [95] proposed a two-step solution for the optimization of large RISs. The main idea of the proposed approach is to divide the RIS into sub-surfaces, each with an equal number of elements, and optimize each sub-surface in two steps, i.e., offline design step and online optimization step. In the offline design step, a set of different configurations of RIS phaseshifts is generated through the joint optimization of subsurfaces. In the transmission step, the best configuration is selected from the set of configurations generated in the online mode in order to achieve maximum performance. Moreover, the authors in [96] exploited the codebook-based approach proposed by [95] to minimize the transmit power consumption of the RIS-aided SWIPT system by jointly optimizing the transmit beamforming and RIS operating mode selection. The authors designed a low-complexity order algorithm, based on the penalty-based method, SCA, and SDR technique, to obtain the sub-optimal solution for the transmit beamforming and RIS mode assignment. Although the codebook-based approach reduces the complexity of online configuration of RIS phaseshifts, however, it degrades the system performance. Hence, the system designer needs to maintain a balance between the complexity and the performance.

\section{AI-EMPOWERED RIS-ASSISTED NETWORKS}

In wireless communication systems, AI based techniques have been recognized as one of the most suitable computational paradigm for improving the operational efficiency. For instance, AI based solutions have shown significant performance improvements for network security [97], resource management [98], [99], activity forecasting [100], user quality of experience [101] and self-organization capabilities [102]. For RIS-assisted networks, AI based techniques are quite useful to solve the important signal processing issues, such as channel estimation, phase-shift optimization and resource allocation, due to their ability to learn underlying trends between the 
TABLE V: Summary of AI-empowered environmental sensing, channel estimation, resource allocation, and phase-shift optimization in RIS-assisted networks.

\begin{tabular}{llll}
\hline Topic & Ref. & AI technique & Explanation \\
\hline \multirow{3}{*}{ Channel estimation } & {$[85]$} & Deep learning & DDNN-based framework for channel estimation in RIS-assisted mmWave system \\
\cline { 2 - 4 } & {$[86]$} & Deep learning & CNN-based model for channel estimation in RIS-assisted MIMO systems \\
\cline { 2 - 4 } Resource allocation & {$[87]$} & Federated learning & Channel estimation scheme for multi-user RIS assisted massive MIMO systems \\
\cline { 2 - 4 } & {$[88]$} & Deep reinforcement learning & User partitioning and RIS beamforming for the RIS assisted NOMA networks \\
\hline \multirow{3}{*}{ Phase-shift optimization } & {$[89]$} & Supervised learning & Performance enhancement for RIS-assisted mmWave system \\
\cline { 2 - 4 } & {$[90]$} & Deep reinforcement learning & Joint resource optimization to maximize the energy efficiency of RIS-assisted network \\
\cline { 2 - 4 } & {$[91]$} & Deep learning & Phase-shift optimization by training the deep feed forward network \\
\cline { 2 - 4 } & {$[93]$} & Supervised learning & Phase-shift optimization via deep neural network framework \\
\cline { 2 - 4 } & {$[94]$} & Deep reinforcement learning & Phase-shift design via environmental learning \\
\hline
\end{tabular}

operating and efficiency parameters for large search space. The existing literature on AI-empowered RIS-assisted networks is summarized in Table V.

\section{A. AI-empowered Channel Estimation}

The accuracy of channel estimation can be improved by utilizing the AI based techniques. In this regard, in [85], the authors proposed a deep denoising neural network based framework for hybrid RIS architecture, consisting of both the active and passive elements, which enables a comprehensive channel estimation with low training overhead for mmWave RIS assisted systems. The proposed approach achieves considerable normalized mean square error (NMSE) performance even with the limited number of activated elements in each training phase. Similarly, in [86], the authors presented a supervised deep learning approach for channel estimation in RIS assisted MIMO systems. The results demonstrated lower normalized mean square error (NSME) and more robust performance of convolution neural network $(\mathrm{CNN})$ based model as compared to conventional methods. Furthermore, in [87], the authors proposed a federated learning based framework for channel estimation in RIS-assisted massive MIMO systems. In the proposed framework, the neural network is trained at the users, rather than the BS. The authors demonstrated that the proposed federated learning framework outperforms the centralized learning-based training in terms of training overhead while achieving the satisfactory channel estimation performance.

\section{B. AI-empowered Resource Allocation}

Besides the channel estimation, the recent research studies have revealed the supremacy of AI based techniques for resource allocation in RIS-assisted networks. For instance,in [88], the authors jointly optimize the user clustering and phases shifts for maximizing the sum rate of RIS-assisted NOMA network. The authors proposed a modified object migration automation (MOMA) algorithm for user clustering and a deep deterministic policy gradient (DDPG) algorithm to optimize the RIS phase-shifts. The results demonstrated the improvement in sum rate by increasing the number of reflecting elements and the granularity of the phase-shifts.
Furthermore, in [89], the authors proposed a mobility and environmentally aware DNN based solution for beam management in RIS-assisted mmWave system. In addition, in [90], the authors proposed a DRL based approach to jointly optimize the power allocation, phase-shifts, and ON/OFF state of the RIS elements, in order to maximize the energy efficiency of RIS-assisted system.

\section{AI-empowered Phase-Shift Optimization}

In [91], the authors proposed a DL based approach for RIS phase reconfiguration, so that it could learn and make usage of local propagation environment. This framework uses received pilot signals to train deep feed forward network, which are reflected through RIS. This trained feed forward DNN can learn how to configure downlink beamforming vector and RIS phase-shifts. In [92], the DL approach was used to learn the reflecting matrices of RIS, directly from sampled channel knowledge, without any knowledge about the geometry array of RIS. Moreover, in [93], the authors proposed a deep neural network (DNN) based design for the reconfiguration of RIS elements, where the optimal phase-shifts are acquired by maximizing the received signal power via trained DNN to map the user's location and element's configurations. Furthermore, in [94], the authors considered a joint optimization of transmit beamforming and RIS phase-shifts by using deep reinforcement learning (DRL) for RIS-assisted massive MIMO system, where one agent drives the best solution through trial-anderror interaction with the environment, and then obtain the RIS phase-shifts.

\section{RESEARCH DiRECTIONS}

Despite the recent advances, the research on RIS-assisted networks is still in infancy. There are several open problems and challenges that are worthy of investigation. Therefore, in this section, we highlight some of the important research directions to spark the future research.

\section{A. Hardware and Channel Imperfections}

Most of the existing works on RIS-assisted networks consider the ideal scenarios, neglecting the hardware and channel 
imperfections. However, in practical RIS-assisted systems, hardware and channel imperfections can be the major performance limitations. Therefore, it is necessary to take these factors into account while evaluating the system performance. Moreover, it is worthwhile to investigate the performance loss caused by these imperfections.

\section{B. Mobility Management}

With the extensive usage of smart devices, number of mobile users are rapidly growing, therefore, an efficient handover management process is a major necessity. Although RIS is a promising technology to mitigate the issues of conventional wireless networks, however, handover management remains an open challenge. Since RIS is passive, it can neither predict nor track the mobility of roaming user by sending pilot signals. The challenge becomes more critical in the absence LoS or direct links between the BS and mobile users, due to the presence of obstacles or network sparsity. Therefore, mobility or handover management in RIS assisted wireless networks is worthy of investigation.

\section{RIS-assisted D2D communication}

D2D communication for the future IoT networks, connecting a massive number of low-power devices, poses more challenges than the conventional BS to receiver communication. This brings new research problems for RIS-assisted D2D communication. Although the capability of RIS to introduce intelligent phase-shifts can enhance the performance of each D2D link, however, the interaction between the RIS and the IoT devices should be kept minimal due to the low operating power of IoT devices. This creates another optimization challenge needing thorough research efforts.

\section{Experimental Validation and Test Beds}

Besides the theoretical analysis, it is important to evaluate the performance of RIS-assisted networks on the experimental grounds to be fully accepted by the wireless community. Although, a few promising experimental activities to support the potential gains offered by RISs have been reported recently. However, these experiments are not sufficient to conclude the potentials of RISs. Therefore, to fully validate the potentials of RISs, it is important to evaluate the performance of RISassisted networks, both theoretically and experimentally, under realistic operating conditions.

\section{CONCLUSION}

In this paper, we comprehensively and systematically surveyed the state-of-the-art on RISs, which are recognized as effective solution for $6 \mathrm{G}$ wireless networks. We first outlined the fundamentals of RIS, with focus on their implementation, hardware architecture, control mechanism, and deployment strategies. Motivated by the compatibility of RIS with other communication technologies, we discussed the emerging applications of RIS in mmWaves and THz communication, MEC networks, UAV-terrestrial networks, vehicular communication and IoT networks. Moreover, we outlined the recent research contributions on RIS CSI acquisition and passive beamforming design. The effectiveness of amalgamating AI and RIS is also highlighted. Finally, to provide effective guidance for future research, we identified the important challenges and open research directions. It is hoped that this paper will serve as useful source for future research to unlock the full potentials of RISs for $6 \mathrm{G}$ wireless networks.

\section{ACKNOWLEDGMENTS}

This work is supported by the EPSRC, UK EP/S016813/1, EP/N010523/1, and Royal Academy of Engineering, UK 122040 grants

\section{REFERENCES}

[1] Miraz, M.H., Ali, M., Excell, P.S., Picking, R.: 'A Review on Internet of Things (IoT), Internet of Everything (IoE) and Internet of Nano Things (IoNT)', 2015 Internet Technologies and Applications (ITA), 2015, pp. 219-224

[2] Chowdhury, M.Z., Shahjalal, M., Ahmed, S., Jang, Y.M.: '6G Wireless Communication Systems: Applications, Requirements, Technologies, Challenges, and Research Directions', IEEE Open Journal of the Communications Society, 2020, 1, pp. 957-975

[3] CISCO: 'Cisco Visual Networking Index: Global Mobile Data Traffic Forecast Update 2017-2022', CISCO, Feb 2019,

[4] Lien, S., Shieh, S., Huang, Y., Su, B., Hsu, Y., Wei, H.: '5G New Radio: Waveform, Frame Structure, Multiple Access, and Initial Access', IEEE Communications Magazine, 2017, 55, (6), pp. 64-71

[5] Andrews, J.G., Bai, T., Kulkarni, M.N., Alkhateeb, A., Gupta, A.K., Heath, R.W.: 'Modeling and Analyzing Millimeter Wave Cellular Systems', IEEE Transactions on Communications, 2017, 65, (1), pp. 403 430

[6] Ngo, H.Q., Larsson, E.G., Marzetta, T.L.: 'Energy and Spectral Efficiency of Very Large Multiuser MIMO Systems', IEEE Transactions on Communications, 2013, 61, (4), pp. 1436-1449

[7] Hashmi, U.S., Zaidi, S.A.R., Darbandi, A., Imran, A.: 'On the Efficiency Tradeoffs in User-Centric Cloud RAN', 2018 IEEE International Conference on Communications (ICC), 2018, pp. 1-7

[8] Hashmi, U.S., Zaidi, S.A.R., Imran, A., Abu-Dayya, A.: 'Enhancing Downlink QoS and Energy Efficiency Through a User-Centric Stienen Cell Architecture for mmWave Networks', IEEE Transactions on Green Communications and Networking, 2020, 4, (2), pp. 387-403

[9] Di Renzo, M., Zappone, A., Merouane, D., Alouini, M.S., Yuen, C., de Rosny, J., et al.: 'Smart Radio Environments Empowered By Reconfigurable Intelligent Surfaces: How It Works, State Of Research, And Road Ahead', arXiv preprint, 2020, Available from: https://arxiv.org/abs/2004.09352

[10] Basharat, S., Hassan, S.A., Pervaiz, H., Mahmood, A., Ding, Z. Gidlund, M.: 'Reconfigurable Intelligent Surfaces: Potentials, Applications, and Challenges for 6G Wireless Networks', IEEE Wireless Communications, 2021, 28, (6), pp. 184-191

[11] Basar, E., Di Renzo, M., De Rosny, J., Debbah, M., Alouini, M., Zhang, R.: 'Wireless Communications Through Reconfigurable Intelligent Surfaces', IEEE Access, 2019, 7, pp. 116753-116773

[12] Wu, Q., Zhang, R.: 'Towards Smart and Reconfigurable Environment: Intelligent Reflecting Surface Aided Wireless Network', IEEE Communications Magazine, 2020, 58, (1), pp. 106-112

[13] Cetinkaya, S., Hashmi, U.S., Imran, A.: 'What User-Cell Association Algorithms will Perform Best in mmWave Massive MIMO Ultradense HetNets?', 2017 IEEE 28th Annual International Symposium on Personal, Indoor, and Mobile Radio Communications (PIMRC), 2017, pp. 1-7

[14] Ni, W., Liu, X., Liu, Y., Tian, H., Chen, Y.: 'Intelligent Reflecting Surface Aided Multi-Cell NOMA Networks', arXiv preprint, 2020, Available from: https://arxiv.org/abs/2012.03611

[15] Sena, A.S.D., Carrillo, D., Fang, F., Nardelli, P.H.J., Costa, D.B.D., Dias, U.S., et al.: 'What Role Do Intelligent Reflecting Surfaces Play in Multi-Antenna Non-Orthogonal Multiple Access?', IEEE Wireless Communications, 2020, 27, (5), pp. 24-31

[16] Yang, G., Xu, X., Liang, Y.C., Di Renzo, M.: 'Reconfigurable Intelligent Surface Assisted Non-Orthogonal Multiple Access', IEEE Transactions on Wireless Communications, 2021, pp. 1-1 
[17] Liu, Y., Mu, X., Liu, X., Di.Renzo, M., Ding, Z., Schober, R.: 'Reconfigurable Intelligent Surface (RIS) Aided Multi-User Networks: Interplay Between NOMA and RIS', arXiv preprint, 2020, Available from: https://arxiv.org/abs/2011.13336

[18] Tan, X., Sun, Z., Koutsonikolas, D., Jornet, J.M.: 'Enabling Indoor Mobile Millimeter-wave Networks Based on Smart Reflect-arrays', IEEE INFOCOM 2018 - IEEE Conference on Computer Communications, 2018, pp. 270-278

[19] Perović, N.S., Renzo, M.D., Flanagan, M.F.: 'Channel Capacity Optimization Using Reconfigurable Intelligent Surfaces in Indoor mmWave Environments', ICC 2020 - 2020 IEEE International Conference on Communications (ICC), 2020, pp. 1-7

[20] Wang, P., Fang, J., Yuan, X., Chen, Z., Li, H.: 'Intelligent Reflecting Surface-Assisted Millimeter Wave Communications: Joint Active and Passive Precoding Design', IEEE Transactions on Vehicular Technology, 2020, 69, (12), pp. 14960-14973

[21] Pan, Y., Wang, K., Pan, C., Zhu, H., Wang, J.: 'Sum Rate Maximization for Intelligent Reflecting Surface assisted Terahertz Communications', arXiv preprint arXiv:200812246, 2020,

[22] Wu, Q., Zhang, Y., Huang, C., Chau, Y., Yang, Z., Shikh.Bahaei, M.: 'Energy Efficient Intelligent Reflecting Surface Assisted Terahertz Communications', 2021 IEEE International Conference on Communications Workshops (ICC Workshops), 2021, pp. 1-6

[23] Bai, T., Pan, C., Deng, Y., Elkashlan, M., Nallanathan, A., Hanzo, L.: 'Latency Minimization for Intelligent Reflecting Surface Aided Mobile Edge Computing', IEEE Journal on Selected Areas in Communications, 2020, 38, (11), pp. 2666-2682

[24] Chu, Z., Xiao, P., Shojafar, M., Mi, D., Mao, J., Hao, W.: 'Intelligent Reflecting Surface Assisted Mobile Edge Computing for Internet of Things', IEEE Wireless Communications Letters, 2020, pp. 1-1

[25] Bai, T., Pan, C., Ren, H., Deng, Y., Elkashlan, M., Nallanathan, A.: 'Resource Allocation for Intelligent Reflecting Surface Aided Wireless Powered Mobile Edge Computing in OFDM Systems', IEEE Transactions on Wireless Communications, 2021,

[26] Liu, Y., Zhao, J., Xiong, Z., Niyato, D., Yuen, C., Pan, C., et al.: 'Intelligent Reflecting Surface Meets Mobile Edge Computing: Enhancing Wireless Communications for Computation Offloading', arXiv preprint, 2020, Available from: https://arxiv.org/pdf/2001.07449.pdf

[27] Huang, S., Wang, S., Wang, R., Wen, M., Huang, K.: 'Reconfigurable Intelligent Surface Assisted Mobile Edge Computing with Heterogeneous Learning Tasks', arXiv preprint, 2020, Available from: https://arxiv.org/abs/2012.13533

[28] Hua, S., Shi, Y.: 'Reconfigurable Intelligent Surface for Green Edge Inference in Machine Learning', 2019 IEEE Globecom Workshops (GC Wkshps), 2019, pp. 1-6

[29] Pan, C., Ren, H., Wang, K., Elkashlan, M., Nallanathan, A., Wang, J., et al.: 'Intelligent Reflecting Surface Aided MIMO Broadcasting for Simultaneous Wireless Information and Power Transfer', IEEE Journal on Selected Areas in Communications, 2020, 38, (8), pp. 1719-1734

[30] Chen, Y., Wang, Y., Zhang, J., Li, Z.: 'Resource Allocation for Intelligent Reflecting Surface Aided Vehicular Communications', IEEE Transactions on Vehicular Technology, 2020, 69, (10), pp. 1232112326

[31] Ozcan, Y.U., Ozdemir, O., Kurt, G.K.: 'Reconfigurable Intelligent Surfaces for the Connectivity of Autonomous Vehicles', arXiv preprint, 2020, Available from: https://arxiv.org/abs/2007.10028

[32] Masini, B., Silva, C., Balador, A.: 'The Use of Meta-Surfaces in Vehicular Networks', Journal of Sensor and Actuator Networks, 2020, 9

[33] Zhang, Q., Saad, W., Bennis, M.: 'Reflections In The Sky: Millimeter Wave Communication With UAV-Carried Intelligent Reflectors', 2019 IEEE Global Communications Conference (GLOBECOM), 2019, pp. $1-6$

[34] Ge, L., Dong, P., Zhang, H., Wang, J.B., You, X.: 'Joint Beamforming and Trajectory Optimization For Intelligent Reflecting SurfacesAssisted UAV Communications', IEEE Access, 2020, 8, pp. 78702 78712

[35] Li, S., Duo, B., Yuan, X., Y..C..Liang, Y.C., Di Renzo, M.: 'Reconfigurable Intelligent Surface Assisted UAV Communication: Joint Trajectory Design and Passive Beamforming', IEEE Wireless Communications Letters, 2020, 9, (5), pp. 716-720

[36] Ma, D., Ding, M., Hassan, M.: 'Enhancing Cellular Communications For UAVs Via Intelligent Reflective Surface', 2020 IEEE Wireless Communications and Networking Conference (WCNC), 2020, pp. 1-6

[37] Almohamad, A., Tahir, A.M., Al-Kababji, A., Furqan, H.M., Khattab, T., Hasna, M.O., et al.: 'Smart and Secure Wireless Communications via Reflecting Intelligent Surfaces: A Short Survey', IEEE Open Journal of the Communications Society, 2020, 1, pp. 1442-1456

[38] Perović, N.S., Tran, L.N., Di.Renzo, M., Flanagan, M.F.: 'Achievable Rate Optimization for MIMO Systems With Reconfigurable Intelligent Surfaces', IEEE Transactions on Wireless Communications, 2021, 20, (6), pp. 3865-3882

[39] Liaskos, C., Nie, S., Tsioliaridou, A., Pitsillides, A., Ioannidis, S. Akyildiz, I.: 'A New Wireless Communication Paradigm through Software-Controlled Metasurfaces', IEEE Communications Magazine, 2018, 56, (9), pp. 162-169

[40] Zhao, J.: 'A Survey of Intelligent Reflecting Surfaces (IRSs): Towards 6G Wireless Communication Networks', arXiv, 2019, abs/1907.04789

[41] Gong, S., Lu, X., Hoang, D.T., Niyato, D., Shu, L., Kim, D.I., et al.: 'Toward Smart Wireless Communications via Intelligent Reflecting Surfaces: A Contemporary Survey', IEEE Communications Surveys Tutorials, 2020, 22, (4), pp. 2283-2314

[42] ElMossallamy, M.A., Zhang, H., Song, L., Seddik, K.G., Han, Z., Li, G.Y.: 'Reconfigurable Intelligent Surfaces for Wireless Communications: Principles, Challenges, and Opportunities', IEEE Transactions on Cognitive Communications and Networking, 2020, 6, (3), pp. 9901002

[43] Liu, Y., Liu, X., Mu, X., Hou, T., Xu, J., Qin, Z., et al.: 'Reconfigurable Intelligent Surfaces: Principles and Opportunities', arXiv preprint, 2020, Available from: https://arxiv.org/abs/2007.03435

[44] Elbir, A.M., Mishra, K.V.: 'A Survey of Deep Learning Architectures for Intelligent Reflecting Surfaces', arXiv preprint, 2020, Available from: https://arxiv.org/abs/2009.02540

[45] Yuan, X., Zhang, Y.J., Shi, Y., Yan, W., Liu, H.: 'ReconfigurableIntelligent-Surface Empowered Wireless Communications: Challenges and Opportunities', IEEE Wireless Communications, 2021, 28, pp. 136143

[46] Di.Renzo, M., Ntontin, K., Song, J., Danufane, F.H., Qian, X., Lazarakis, F., et al.: 'Reconfigurable Intelligent Surfaces vs. Relaying: Differences, Similarities, and Performance Comparison', IEEE Open Journal of the Communications Society, 2020, 1, pp. 798-807

[47] Wu, Q., Zhang, S., Zheng, B., You, C., Zhang, R.: 'Intelligent Reflecting Surface Aided Wireless Communications: A Tutorial', IEEE Transactions on Communications, 2021, pp. 1-1

[48] Wei, X., Shen, D., Dai, L.: 'Channel Estimation for RIS Assisted Wireless Communications-Part I: Fundamentals, Solutions, and Future Opportunities', IEEE Communications Letters, 2021, 25, (5), pp. 13981402

[49] Yu, X., Jamali, V., Xu, D., Ng, D.W.K., Schober, R.: 'Smart and Reconfigurable Wireless Communications: From IRS Modeling to Algorithm Design', ArXiv, 2021, abs/2103.07046

[50] Basharat, S., Hassan, S.A., Mahmood, A., Ding, Z., Gidlund, M.: 'Reconfigurable Intelligent Surface-Assisted Backscatter Communication: A New Frontier for Enabling 6G IoT Networks', ArXiv, 2021, abs/2107.07813

[51] Chen, H.T., Taylor, A.J., Yu, N.: 'A Review Of Metasurfaces: Physics and Applications', Reports on progress in physics, 2016, 79, (7), pp. 076401

[52] Cui, T., Qi, M., Wan, X., Zhao, J., Cheng, Q.: 'Coding Metamaterials, Digital Metamaterials and Programmable Metamaterials', LightScience \& Applications, 2014, 3

[53] Subrt, L., Pechac, P.: 'Controlling Propagation Environments using Intelligent Walls', 2012 6th European Conference on Antennas and Propagation (EUCAP), 2012, pp. 1-5

[54] Hum, S.V., Perruisseau.Carrier, J.: 'Reconfigurable Reflectarrays and Array Lenses for Dynamic Antenna Beam Control: A Review', IEEE Transactions on Antennas and Propagation, 2014, 62, (1), pp. 183-198

[55] Foo, S.: 'Liquid-Crystal Reconfigurable Metasurface Reflectors', 2017 IEEE International Symposium on Antennas and Propagation USNC/URSI National Radio Science Meeting, 2017, pp. 2069-2070

[56] Yang, Z., Chen, M., Saad, W., Xu, W., Shikh-Bahaei, M., Poor, H.V., et al.: 'Resource Allocation for Wireless Communications with Distributed Reconfigurable Intelligent Surfaces', 2020 IEEE Global Communications Conference, 2020, pp. 1-6

[57] You, C., Zheng, B., Zhang, R.: 'How to Deploy Intelligent Reflecting Surfaces in Wireless Network: BS-side, User-side, or Both Sides?', arXiv preprint, 2020, Available from: https://arxiv.org/abs/2012.03403

[58] Lu, H., Zeng, Y., Jin, S., Zhang, R.: 'Aerial Intelligent Reflecting Surface: Joint Placement and Passive Beamforming Design with 3D Beam Flattening', arXiv preprint, 2020, Available from: https://arxiv. org/abs/2007.13295

[59] Park, S.Y., In Kim, D.: 'Intelligent Reflecting Surface-aided Phase-Shift Backscatter Communication', 2020 14th International Conference on 
Ubiquitous Information Management and Communication (IMCOM), 2020, pp. $1-5$

[60] Chu, Z., Xiao, P., Mi, D., Chen, H., Hao, W.: 'Intelligent Reflecting Surfaces Enabled Cognitive Internet of Things Based on Practical Pathloss Model', China Communications, 2020, 17, (12), pp. 1-16

[61] Guo, Y., Qin, Z., Liu, Y., Al-Dhahir, N.: 'Intelligent Reflecting Surface Aided Multiple Access Over Fading Channels', IEEE Transactions on Communications, 2020, pp. 1-1

[62] Zhang, L., Chen, X.Q., Liu, S., Zhang, Q., Zhao, J., Dai, J.Y., et al.: 'Space-Time-Coding Digital Metasurfaces', 2019 Thirteenth International Congress on Artificial Materials for Novel Wave Phenomena (Metamaterials), 2019, pp. X-128-X-130

[63] Lu, H., Zeng, Y., Jin, S., Zhang, R.: 'Enabling Panoramic Full-Angle Reflection Via Aerial Intelligent Reflecting Surface', 2020 IEEE International Conference on Communications Workshops (ICC Workshops), 2020, pp. 1-6

[64] Hartmann, M., Hashmi, U.S., Imran, A.: 'Edge Computing in Smart Health Care Systems: Review, Challenges, and Research Directions', Transactions on Emerging Telecommunications Technologies, 2019, Available from: https://onlinelibrary.wiley.com/doi/abs/10.1002/ ett. 3710

[65] Zhao, M.M., Shi, Q., Zhao, M.J.: 'Efficiency Maximization for UAVenabled Mobile Relaying Systems With Laser Charging', IEEE Transactions on Wireless Communications, 2020, 19, (5), pp. 3257-3272

[66] Zeng, Y., Zhang, R., Lim, T.J.: 'Wireless Communications With Unmanned Aerial Vehicles: Opportunities and Challenges', IEEE Communications Magazine, 2016, 54, (5), pp. 36-42

[67] Wu, Q., Zhang, R.: 'Intelligent Reflecting Surface Enhanced Wireless Network via Joint Active and Passive Beamforming', IEEE Transactions on Wireless Communications, 2019, 18, (11), pp. 5394-5409

[68] Mishra, D., Johansson, H.: ; IEEE. 'Channel Estimation and Lowcomplexity Beamforming Design for Passive Intelligent Surface Assisted MISO Wireless Energy Transfer', ICASSP 2019-2019 IEEE International Conference on Acoustics, Speech and Signal Processing (ICASSP), 2019, pp. 4659-4663

[69] Yang, Y., Zheng, B., Zhang, S., Zhang, R.: 'Intelligent Reflecting Surface Meets OFDM: Protocol Design and Rate Maximization', IEEE Transactions on Communications, 2020, 68, (7), pp. 4522-4535

[70] Nadeem, Q.U.A., Alwazani, H., Kammoun, A., Chaaban, A., Debbah, M., Alouini, M.S.: 'Intelligent Reflecting Surface-Assisted Multi-User MISO Communication: Channel Estimation and Beamforming Design', IEEE Open Journal of the Communications Society, 2020, 1, pp. 661680

[71] Guan, X., Wu, Q., Zhang, R.: 'Anchor-assisted Channel Estimation for Intelligent Reflecting Surface Aided Multiuser Communication', ArXiv, 2021, abs/2102.10886

[72] Wang, P., Fang, J., Duan, H., Li, H.: 'Compressed Channel Estimation for Intelligent Reflecting Surface-Assisted Millimeter Wave Systems', IEEE Signal Processing Letters, 2020, 27, pp. 905-909

[73] Chen, J., Liang, Y.C., Cheng, H.V., Yu, W.: 'Channel estimation for Reconfigurable Intelligent Surface Aided Multi-user MIMO Systems', arXiv, 2019, abs/1912.03619

[74] Wu, Q., Zhang, R.: 'Intelligent Reflecting Surface Enhanced Wireless Network via Joint Active and Passive Beamforming', IEEE Transactions on Wireless Communications, 2019, 18, (11), pp. 5394-5409

[75] Wu, Q., Zhang, R.: 'Beamforming Optimization for Wireless Network Aided by Intelligent Reflecting Surface With Discrete Phase Shifts', IEEE Transactions on Communications, 2019, 68, (3), pp. 1838-1851

[76] Yang, Y., Zhang, S., Zhang, R.: 'IRS-Enhanced OFDM: Power Allocation and Passive Array Optimization', 2019 IEEE Global Communications Conference (GLOBECOM), 2019, pp. 1-6

[77] Guo, H., Liang, Y.C., Chen, J., Larsson, E.G.: 'Weighted Sum-Rate Maximization for Intelligent Reflecting Surface Enhanced Wireless Networks', 2019 IEEE Global Communications Conference (GLOBECOM), 2019, pp. 1-6

[78] Liu, Y., Zhao, J., Li, M., Wu, Q.: 'Intelligent Reflecting Surface Aided MISO Uplink Communication Network: Feasibility and SINR Optimization', ArXiv, 2020, abs/2007.01482

[79] Huang, C., Zappone, A., Alexandropoulos, G.C., Debbah, M., Yuen, C.: 'Reconfigurable intelligent surfaces for energy efficiency in wireless communication', IEEE Transactions on Wireless Communications, 2019, 18, (8), pp. 4157-4170

[80] Yang, Z., Shi, J., Li, Z., Chen, M., Xu, W., Shikh.Bahaei, M.: 'Energy Efficient Rate Splitting Multiple Access (RSMA) with Reconfigurable Intelligent Surface', 2020 IEEE International Conference on Communications Workshops (ICC Workshops), 2020, pp. 1-6
[81] Liu, Y., Zhao, J., Li, M., Wu, Q.: 'Intelligent Reflecting Surface Aided MISO Uplink Communication Network: Feasibility and Power Minimization for Perfect and Imperfect CSI', IEEE Transactions on Communications, 2021, 69, (3), pp. 1975-1989

[82] Fara, R., Phan-Huy, D., Ratajczak, P., Ourir, A., M..Di Renzo, M., de Rosny, J.: 'Reconfigurable Intelligent Surface-Assisted Ambient Backscatter Communications-Experimental Assessment', arXiv preprint, 2021, Available from: https://arxiv.org/ftp/arxiv/papers/2103/ 2103.08427.pdf

[83] Zheng, B., Zhang, R.: 'Intelligent reflecting surface-enhanced ofdm: Channel estimation and reflection optimization', IEEE Wireless Communications Letters, 2019, 9, (4), pp. 518-522

[84] Jamali, V., Najafi, M., Schober, R., Poor, H.V.: 'Power Efficiency, Overhead, and Complexity Tradeoff in IRS-Assisted CommunicationsQuadratic Phase-Shift Design', arXiv, 2020, abs/2009.05956

[85] Liu, S., Gao, Z., Zhang, J., Renzo, M.D., Alouini, M.S.: 'Deep Denoising Neural Network Assisted Compressive Channel Estimation for mmWave Intelligent Reflecting Surfaces', IEEE Transactions on Vehicular Technology, 2020, 69, (8), pp. 9223-9228

[86] Elbir, A.M., Papazafeiropoulos, A., Kourtessis, P., Chatzinotas, S.: 'Deep Channel Learning For Large Intelligent Surfaces Aided mmWave Massive MIMO Systems', IEEE Wireless Communications Letters, 2020,

[87] Elbir, A.M., Coleri, S.: 'Federated Learning for Channel Estimation in Conventional and IRS-assisted Massive MIMO', arXiv, 2020, abs/2008.10846

[88] Yang, Z., Liu, Y., Chen, Y., Al.Dhahir, N.: 'Machine Learning for User Partitioning and Phase Shifters Design in RIS-Aided NOMA Networks', arXiv preprint arXiv:210101212, 2021,

[89] Jia, C., Gao, H., Chen, N., He, Y.: 'Machine Learning Empowered Beam Management for Intelligent Reflecting Surface Assisted MmWave Networks', China Communications, 2020, 17, (10), pp. 100114

[90] Lee, G., Jung, M., Kasgari, A.T.Z., Saad, W., Bennis, M.: 'Deep Reinforcement Learning for Energy-Efficient Networking with Reconfigurable Intelligent Surfaces', ICC 2020 - 2020 IEEE International Conference on Communications (ICC), 2020, pp. 1-6

[91] Özdogan, Ö., Björnson, E.: 'Deep Learning-based Phase Reconfiguration for Intelligent Reflecting Surfaces', arXiv preprint, 2020, Available from: https://arxiv.org/pdf/2009.13988.pdf

[92] Taha, A., Alrabeiah, M., Alkhateeb, A.: 'Enabling Large Intelligent Surfaces with Compressive Sensing and Deep Learning', arXiv preprint, 2019, Available from: https://arxiv.org/abs/1904.10136

[93] Huang, C., Alexandropoulos, G.C., Yuen, C., Debbah, M.: 'Indoor Signal Focusing with Deep Learning Designed Reconfigurable Intelligent Surfaces', 2019 IEEE 20th International Workshop on Signal Processing Advances in Wireless Communications (SPAWC), 2019, pp. $1-5$

[94] Huang, C., Mo, R., Yuen, C.: 'Reconfigurable Intelligent Surface Assisted Multiuser MISO Systems Exploiting Deep Reinforcement Learning', IEEE Journal on Selected Areas in Communications, 2020, 38, (8), pp. 1839-1850

[95] Najafi, M., Jamali, V., Schober, R., Poor, H.V.: 'Physics-based Modeling and Scalable Optimization of Large Intelligent Reflecting Surfaces', IEEE Transactions on Communications, 2020, 69, (4), pp. 2673-2691

[96] Xu, D., Yu, X., Jamali, V., Ng, D.W.K., Schober, R.: 'Resource Allocation for Large IRS-Assisted SWIPT Systems with Non-linear Energy Harvesting Model', 2021 IEEE Wireless Communications and Networking Conference (WCNC), 2021, pp. 1-7

[97] Hussain, F., Hussain, R., Hassan, S.A., Hossain, E.: 'Machine Learning in IoT Security: Current Solutions and Future Challenges', IEEE Communications Surveys Tutorials, 2020, 22, (3), pp. 1686-1721

[98] Shaghaghi, M., Adve, R.S.: 'Machine Learning Based Cognitive Radar Resource Management', 2018 IEEE Radar Conference (RadarConf18), 2018, pp. 1433-1438

[99] Ibrahim, M., Hashmi, U.S., Nabeel, M., Imran, A., Ekin, S.: 'Embracing Complexity: Agent-Based Modeling for HetNets Design and Optimization via Concurrent Reinforcement Learning Algorithms', IEEE Transactions on Network and Service Management, 2021, 18, (4), pp. 4042-4062

[100] Naveed, M.H., Hashmi, U.S., Tajved, N., Sultan, N., Imran, A.. 'Is Synthetic The New Real? Performance Analysis of Time Series Generation Techniques with Focus on Network Load Forecasting'. (TechRxiv, 2021

[101] Hashmi, U.S., Rudrapatna, A., Zhao, Z., Rozwadowski, M., Kang, J., Wuppalapati, R., et al.: 'Towards Real-Time User QoE Assessment via 
Machine Learning on LTE Network Data', 2019 IEEE 90th Vehicular Technology Conference (VTC2019-Fall), 2019, pp. 1-7

[102] Hashmi, U.S., Darbandi, A., Imran, A.: 'Enabling Proactive SelfHealing by Data Mining Network Failure Logs', 2017 International Conference on Computing, Networking and Communications (ICNC), 2017, pp. 511-517 